\title{
TESTING OF YARN ABRASION
}

\author{
Krupincová, G. ${ }^{1}$, Hatipoglu, J. ${ }^{2}$ \\ ${ }^{1}$ Technical University of Liberec, Department of Textile Technology, Liberec, Czech Republic \\ Tel.: +420 48535 342474, Fax: +420 48535 3542, E-mail: gabriela.krupincova@tul.cz, \\ ²Ege University, Textile Engineering Department, Izmir, Turkey, E-mail: yakuphatipoglu@gmail.com
}

\begin{abstract}
:
There exist a lot of methodologies, which can be used for yarn quality testing. Abrasion resistance and its measurement for raw and sized yarn can help in the judgment of yarn weaving-ability. This article concentrates on the possibility of yarn abrasion expression and testing. Relation among fiber material characteristics, selected yarn structural, and mechanical parameters is discussed and a few experimental results are shown.
\end{abstract}

\section{Keywords:}

Structural and mechanical yarn characteristics, yarn abrasion resistance.

\section{Introduction}

Higher production and higher demands of customers on fabric utility value means higher requirements on yarn production quality. Selected fiber material, chosen spinning technology, and all previous operations are necessary to be applied before weaving, since their correct application influences fabric and end-product quality. Performance of warp yarns on a loom during weaving is affected by a number of factors as it is subjected to complex deformation including abrasion, cyclic bending together with tension and impact loading. Controlling yarn's structural characteristics and examining of level of mechanical parameters together with evaluation of yarn's weaving-ability is essential. Abrasion resistance and its measurement for raw and sized yarn can help in the judgment of yarn's weaving-ability.

\section{Yarn abrasion}

\subsection{Testing possibilities}

Methodologies used for yarn abrasion resistance testing can be divided into two groups. First group uses a defined abrasion material. Results from this test are comparable [7]. An example of this instrument is Zweigle G 552 tester [2], Wira tester [5] or CTT yarn abrasion tester [9]. Simulation of mechanical behavior on laboratory loom or on its function parts can give results more close to real weaving. On the other hand simulation is limited too. Loom settings, its speed, all interaction among yarns and guiding places are different for various kinds of looms. Representative instrument is Reutlinger Webtester [1]. Simulation of "yarn on yarn" abrasion can help us in understanding the mechanism of yarn damage during yarn on yarn contact. Staff tester of Zweigle [5] simulates the running characteristics of spun yarns and smooth plied yarns. Specia method "yarn on yarn" is used for testing of rope or specific yarns made from special synthetic fibers. Measurement can be realized in normal or wet conditions [8].
Zweigle G 522 method was used during the experiment and therefore few things need to be addressed. Usually up to twenty threads are placed in the abrasion tester; thereafter pre-tension is applied (usually $20 \mathrm{~g}$ or $30 \mathrm{~g}$ per thread). Everything proceeds automatically: An abrasion roller covered with emery paper traverses in constant rhythmic motion and constant pressure at right angles to the direction at which the test threads are tensioned. The abrasion roller continuously rotates about its own axis so that an abrasive action is not impaired by abraded yarn residue in the emery paper. The computer controls the test procedure and logs the yarn breaks. Special optical sensors are activated when a weight drops down, and number of strokes for all samples is recorded in a database [2].

\subsection{Approaches to yarn abrasion description}

Abrasion resistance is usually expressed as a number of strokes to yarn destruction. A criterion based on weight reduction is a bit problematic, because limited yarn length is possible to weight. A weight reduction due to abrasion can be easy described thanks to yarn diameter changes. The diameters of original yarn samples and yarns after extension of $50 \%$ of the number of strokes for yarn destruction can be observed according to internal methodology IN 32-102-01/01 [10].

The method is based on scanning and processing images of yarn longitudinal views. Color images are transformed through gray-scale to binary images by using Otsu's method [4]. Fibers that belong to the hairiness sphere are eliminated by morphological operation (erosion, dilatation, opening, and closing of image). All image rows are processed step by step. Number of pixels belonging to yarn is counted. Their original length is recalculated by used calibration. Evaluation of each image row - potential yarn diameter (original yarn diameter $D$, yarn diameter after abrasion $D a$ ), outlier values exclusion, statistical yarn diameter finding - follows. Yarn diameter as a mean value itself cannot describe diameter change completely. Minimum $D a_{\min }$, maximum $D a_{\max }$, and mean value $D a$ of yarn rows' length can qualify yarn dimensions after abrasion. 
Minimum diameter means the shortest row length of imaginary yarn cross-section. Maximum diameter means diameter of cylinder, which can cover the yarn. In other words, it is a difference between the smallest coordinate and the highest coordinate, where black pixel that belongs to yarn is placed in hole image and not only in actual image row. The percentage change of yarn diameter before and after abrasion can express abrasion resistance.

\section{EXPERIMENT}

The influence of selected factors on abrasion resistance is investigated. The level of influencing factors is evaluated thanks to correlation and ANOVA analysis. Pair and partial correlation coefficient was used for expression of strength and direction of a linear relationship between two random variables. It was calculated according to eq. (1a,b). Pair correlation measures the strength of the relationship between two random variables. It does not take other influencing factors into consideration. Partial correlation is more sufficient, because it measures the degree of association between two random variables, with the effect of a set of controlling random variables removed. ANOVA analysis enables dividing of variance into different components due to explanatory variables. Two-dimensional ANOVA analysis with fixed-effects model was used for data processing.

$$
\begin{aligned}
& R_{12}=\frac{\operatorname{cov}\left(X_{1}, X_{2}\right)}{\sqrt{\operatorname{var} X_{1} \operatorname{var} \mathrm{X}_{2}}}, \\
& R_{1 i(2,3, \ldots k)}=\frac{(-1)^{i} \operatorname{det}\left(R_{1, i}\right)}{\sqrt{\operatorname{det}\left(R_{11}\right) \operatorname{det}\left(\mathrm{R}_{\mathrm{i}, \mathrm{i}}\right)}} .
\end{aligned}
$$

\section{$\underline{3.1 \text { Experimental material }}$}

The idea of this experiment is to prepare yarns from various fiber materials with similar yarn geometrical parameters under comparable condition. A set of one component and blended single and two-ply ring spun yarns was used for the experiment.

Step 1: One-component single ring spun yarns were spun in five levels of yarn count and three levels of Phrix twist coefficient from $100 \%$ PET, PAN, VS fibers. The information about the fiber material is shown in Table 1. One-component single ring spun yarns are described in Table 2.

Step 2: Set of blended single and two ply yarns were produced with typical yarn count level 29,5 tex, respectively $2 \times 29,5$ tex and optimal Phrix twist coefficient in five level of blending portion of polypropylene PP and cotton CO fibers. The information about the fiber material is shown in Table 1. The description of this set of yarns is given in Table 3 .

Table 1. Selected fiber parameters.

\begin{tabular}{|c|c|c|c|c|c|c|}
\hline & & PET & PAN & VS & CO (Egypt Giza 70) & PP \\
\hline $\boldsymbol{t}_{n} / \boldsymbol{t}_{\mathbf{v}}$ & {$[$ dtex] } & $1,3 / 1,4$ & $0,9 / 1,17$ & $1,3 / 1,34$ & 1,65 & 1,88 \\
\hline & & $(1,36 ; 1,45)$ & $(1,13 ; 1,21)$ & $(1,30 ; 1,37)$ & $(1,53 ; 1,77)$ & $(1,80 ; 1,95)$ \\
\hline$r_{v}$ & {$\left[\mathbf{k g m}^{-3}\right]$} & 1360 & 1170 & 1520 & 1520 & 910 \\
\hline $\boldsymbol{I}$ & {$\left[\mathbf{m m}^{2}\right]$} & 38 & 38 & 38 & 31,13 & 50 \\
\hline $\boldsymbol{R F}$ & {$\left[\mathbf{m N m m}^{2} \mathbf{t e x}^{-1}\right]$} & 0,3 & $0,33-0,48$ & 0,19 & 0,19 & 0,51 \\
\hline $\boldsymbol{f}_{v}$ & {$\left[\mathbf{c N t e x}^{-1}\right]$} & 53,32 & 33,97 & 17,56 & 40,5 & 40,42 \\
\hline & & $(51,67 ; 54,98)$ & $(32,85 ; 35,08)$ & $(16,77 ; 18,34)$ & $(36,6 ; 44,4)$ & $(39,35 ; 41,08)$ \\
\hline $\mathbf{e}_{v}$ & {$[\%]$} & 17,51 & 31,86 & 30,05 & 5,95 & 63,35 \\
\hline & & $(16,27 ; 18,74)$ & $(30,63 ; 33,08)$ & $(29,11 ; 30,99)$ & $(5,41 ; 6,48)$ & $(58,13 ; 68,56)$ \\
\hline
\end{tabular}

\begin{tabular}{|c|c|c|c|c|c|c|c|c|c|c|c|c|c|c|c|}
\hline material & \multicolumn{3}{|c|}{ PET, PAN, VS } & \multicolumn{3}{|c|}{ PET, PAN, VS } & \multicolumn{3}{|c|}{ PET, PAN, VS } & \multicolumn{3}{|c|}{ PET, PAN, VS } & \multicolumn{3}{|c|}{ PET, PAN, VS } \\
\hline$T[$ tex $]$ & \multicolumn{3}{|c|}{16,5} & \multicolumn{3}{|c|}{20} & \multicolumn{3}{|c|}{29,5} & \multicolumn{3}{|c|}{35,5} & \multicolumn{3}{|c|}{42} \\
\hline$a\left[k t^{2 / 3} \mathbf{m}^{-1}\right]$ & 50 & 56 & 62 & 50 & 56 & 62 & 50 & 56 & 62 & 50 & 56 & 62 & 50 & 56 & 62 \\
\hline
\end{tabular}

Table 2. Description of one-component single ring spun yarns.

Table 3. Description of blended single and two-ply yarns.

\begin{tabular}{|c|c|c|c|c|c|c|c|c|c|c|}
\hline material & \multicolumn{2}{|c|}{$\mathbf{1 0 0} \% \mathrm{PP}$} & \multicolumn{2}{c|}{$\mathbf{3 5 \% C O / 6 5 \% P P}$} & \multicolumn{2}{c|}{$\mathbf{5 0 \% C O / \% P P}$} & \multicolumn{2}{c|}{$\mathbf{6 5 \% C O / 3 5 \% P P}$} & \multicolumn{2}{c|}{$100 \% \mathrm{CO}$} \\
\hline & single & two-ply & single & two-ply & single & two-ply & single & two-ply & single & two-ply \\
\hline $\boldsymbol{T}$ [tex] & 29,5 & $2 \times 29,5$ & 29,5 & $2 \times 29,5$ & 29,5 & $2 \times 29,5$ & 29,5 & $2 \times 29,5$ & 29,5 & $2 \times 29,5$ \\
\hline$Z\left[\mathbf{m}^{-1}\right]$ & 601 & 350 & 643 & 359 & 621 & 357 & 632 & 380 & 625 & 371 \\
\hline & $(593 ; 610)$ & $(340 ; 360)$ & $(637 ; 648)$ & $(350 ; 368)$ & $(615 ; 626)$ & $(350 ; 365)$ & $(624 ; 639)$ & $(370 ; 389)$ & $(616 ; 633)$ & $(360 ; 383)$ \\
\hline
\end{tabular}




\subsection{Testing conditions}

Testing of selected fiber and yarn parameters was realized according to the Czech European International Standard. Conditioning of samples was made in respect to ČSN-EN-ISO-2061. Fiber fineness and mechanical parameters were measured according to ČSN-EN-ISO-1973 by Vibroskop \& Vibrodyn instruments (gauche length $10 \mathrm{~mm}$, pretension selected according fibermaterial, 50 measurements). Controlling of yarn count and yarn twist was realized in agreement with ČSN-EN-ISO-2060, ČSN-EN-ISO-2061. Yarn mechanical parameters were tested according to ČSN-EN-ISO-2062 on Instron tester (gauche length $50 \mathrm{~mm}$, pretension selected according yarn count, time of correct test up to $20 \mathrm{~s} \pm 3 \mathrm{~s}, 50$ correct measurements). Zweigle G 552 instrument was used for measuring number of stokes to destruction (pretension $20 \mathrm{~g}$, emery paper $\mathrm{P} 800$ with abrasive grain alpha $\mathrm{Al}_{2} \mathrm{O}_{3}$ and loom reed, 60 measurement). Yarn diameter change due to abrasion was studied only for the set of blended yarns. Diameters of original yarn sample and yarn after extension of $50 \%$ of number of strokes to yarn destruction were observed according to internal methodology IN 32-102-01/01 [10] (image resolution 548pxl x 704pxl, calibration 2,23 $\mathrm{mmpx|}^{-1}$ ).

\section{$\underline{3.3 \text { Discussion of experimental results }}$}

The relation among fiber parameters, yarn count, yarn twist, and mechanical parameters is well known. Theoretical presumptions were confirmed by many experiments. It is generally accepted that yarn strength is related with fiber characteristics (mechanical parameters, stiffness, friction, and flexural rigidity), yarn structural characteristic (count, twist coefficient, and packing density), and technology of production. Production technology selection influences the level of fiber arrangement. Higher degree of fiber arrangement in yarn means better yarn mechanical properties. Correct level of yarn count and twist is important from the point of view of yarn packing density. Higher compactness of fiber in yarn causes stronger utilization of fiber strength and inter-fiber slippage. Using of stronger, stiffer fiber material with higher yarn count and higher twist leads to stronger yarn with lower elongation. It can be expected that similar assumptions will be valid for yarn abrasion.

Simulation of yarn abrasion straining on a loom was realized using Zweigle G 552 tester. Yarn structure was opened during yarn abrasion and twists were pushed to the ends of the testing zone. Bundle of fibers and its damaged segments created entanglements on the sample body (see Figure 1). Inter-fiber slippage supported by pretension and structure opening tended to break the sample. Knowledge of dimension and the occurrence of thin and thick places on yarn's body is important. Thick places create trouble while passing through all the guiding places on a loom. Thin places are a risky part of the chain that increases the warp breakages. It is necessary to mention that the images of yarn are transformed from 3D to $2 \mathrm{D}$ and therefore dimension of thin or thick places is not fully described. An example of yarn longitudinal view of 29,5 tex yarn before and after abrasion due to emery paper is shown in Figure 2 (Figure 2a shows the original yarn sample, Figure $2 b$ the thin place, and Figure 2c thick place of the sample).

Multivariate data analysis (Correlation and ANOVA analysis) was used for data processing. Question of significance of the power of various factors on yarn abrasion resistance was solved in two steps. A set of single yarn samples allows us to judge the influence of fiber material, and structural and mechanical characteristics on abrasion resistance (step 1).

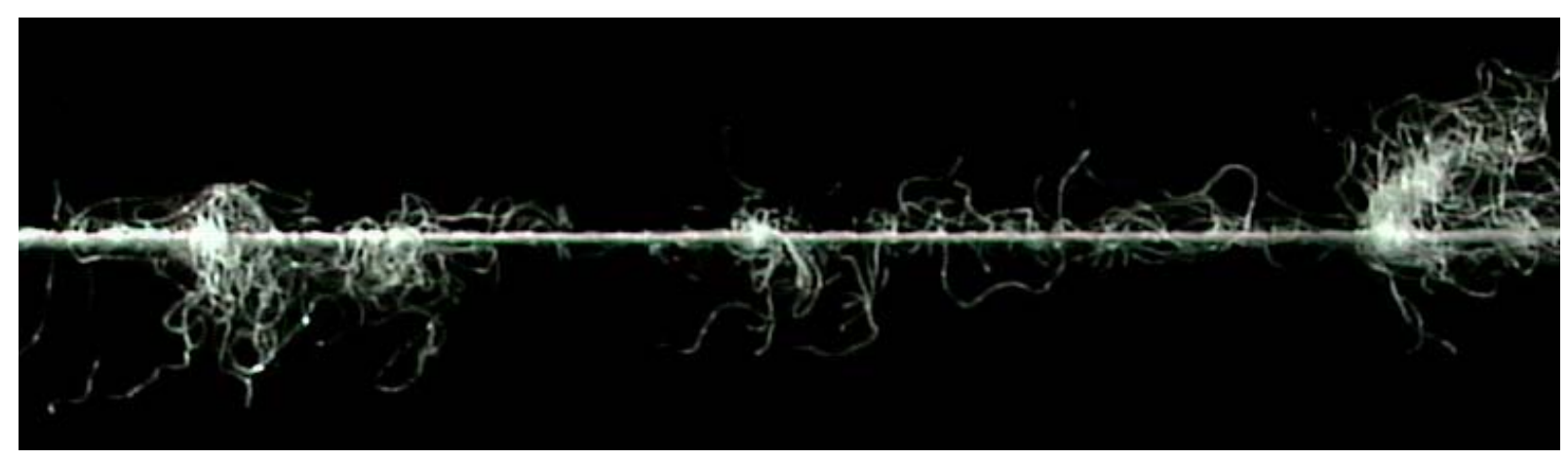

Figure 1. $100 \%$ cotton single yarn 29,5 tex after $50 \%$ of the number of strokes to yarn destruction (calibration $4,72 \mu \mathrm{mpxl}^{-1}$, resolution of image $548 \mathrm{pxl}$ x 704pxl).

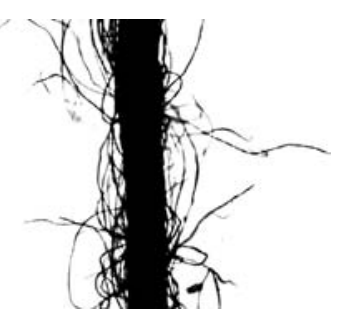

b

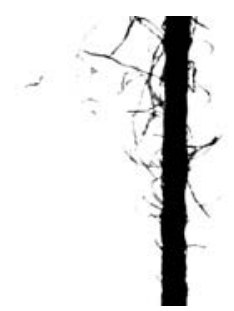

C

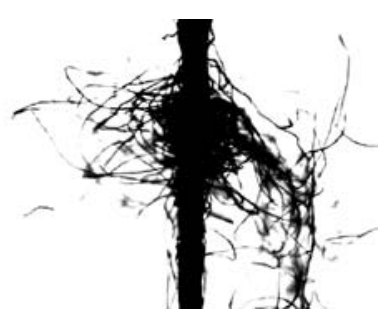

Figure 2. $100 \%$ cotton single yarn 29,5 tex before and after $50 \%$ of the number of strokes to yarn destruction (calibration $2,23 \mu \mathrm{mpx}{ }^{-1}$, resolution of image $548 \mathrm{pxl} \times 704 \mathrm{pxl})$. 
The effect of blending portion together with plying technology on abrasion resistance can be studied, thanks to comparison of blended single and two-ply yarn results (step 2).

Step 1: Influence of selected fiber (fineness $t_{v}$, diameter $d_{v}$, mass density $r_{\mathrm{v}}$, flexural rigidity $R F$, strength $f_{\mathrm{v}}$, and elongation $e_{\mathrm{v}}$ ) and yarn characteristics (nominal count $T_{\mathrm{jm}}$, Phrix twist coefficient $a$, experimental count $T_{\text {exp }}$, twist number $Z$, and mechanical parameters $F, e$ ) on yarn abrasion resistance was investigated for a set of one component single yarns defined in Table 2. Yarn abrasion resistance was expressed as a number of strokes to yarn destruction $a_{1}$. Number of strokes is highly correlated with yarn count. Therefore, the ratio between the number of strokes and yarn count was added to data analysis $a_{2}$. Correlation map for paired correlation coefficients is shown in Figure $3 a$ and for partial correlation coefficients in Figure 3b.

Thanks to multivariate data analysis, it was found that fiber strength, yarn count, yarn strength, and elongation are significantly related to abrasion resistance (paired and partial correlation coefficients higher than 0,5 ). It was verified that number of strokes to yarn destruction $a_{1}$ is positively correlated with yarn count (partial correlation coefficient 0,64 ). Influence of twist level is not as significant as we expected. This approach is limited because of a mutually connected factor (multicolinearity), factor's limited range, and proper selection of technological yarn creation parameters (interdependence yarn count, yarn twist).

Step 2: Set of blended single and two-ply yarns is very interesting, since polypropylene is normally not used for blending with cotton fibers. These two kinds of fibers are dissimilar in many characteristics, but an otherness in mass densities and

a

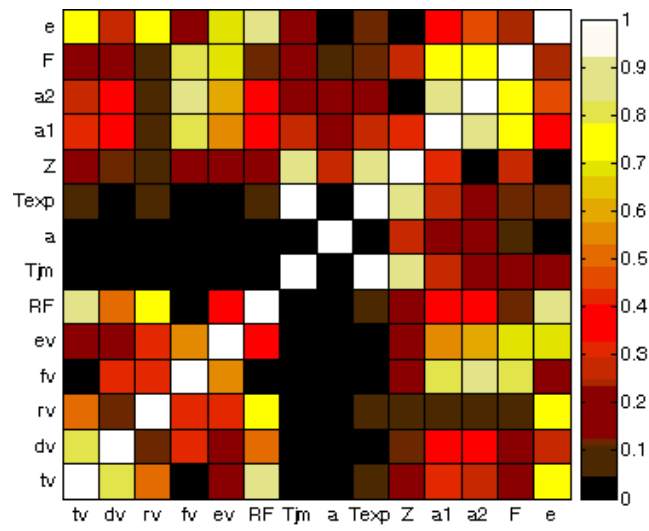

b

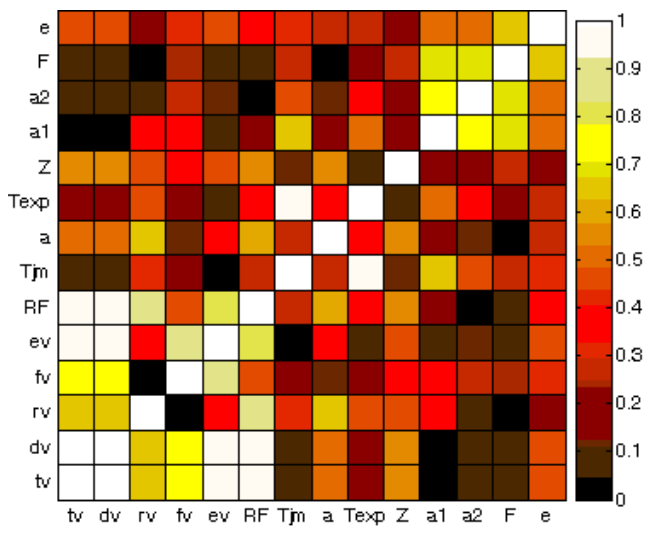

Figure 3. Correlation map for paired correlation coefficients and for partial correlation coefficients (step 1). mechanical parameters give blended yarns attractive parameters. When we use well-known relation among fiber mass density, fibers characteristics (e.g., fineness $t_{v}$, strength $f_{v}$, elongation $\mathrm{e}_{\mathrm{v}}$ ), and yarn's parameters (e.g. count $T$, twist $Z$, tenacity $F$, and elongation $e$ ), we can find interesting connection. Same fineness of cotton and polypropylene fibers means higher diameter of polypropylene fibers. Polypropylene fibers have higher tenacity and elongation than cotton fibers, because of their chemical nature. Same yarn count and same level of twists means higher number of polypropylene fibers in yarn cross-section. This phenomenon leads to higher diameter and higher tenacity of polypropylene yarn. Dependence of yarn tenacity on blending ratio can be predicted by Hamburger's theory [10]. Modeling of other mechanical parameters (e.g., elongation, abrasion resistance) is problematic and exists only in regression equation.

Figure 4a, b, c shows relationships between yarn tenacity, yarn elongation, abrasion resistance, and blending portion of cotton fibers for single and two-ply yarns. The obtained results

a

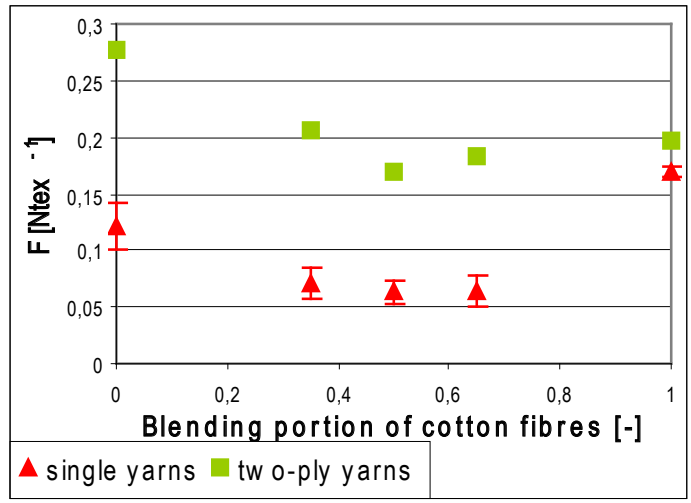

b

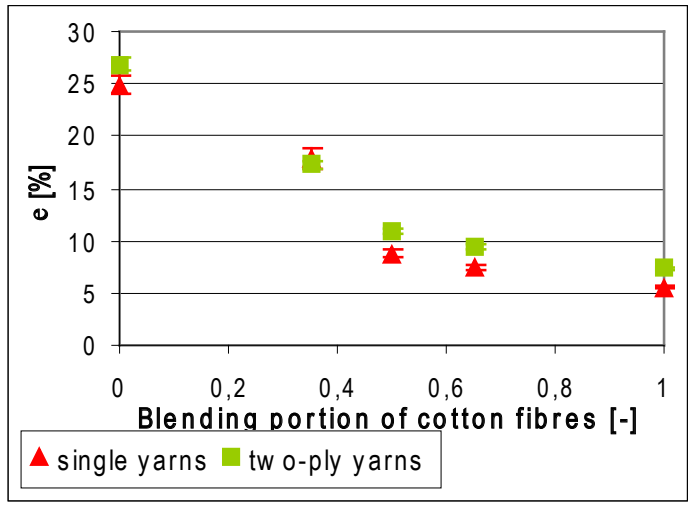

C

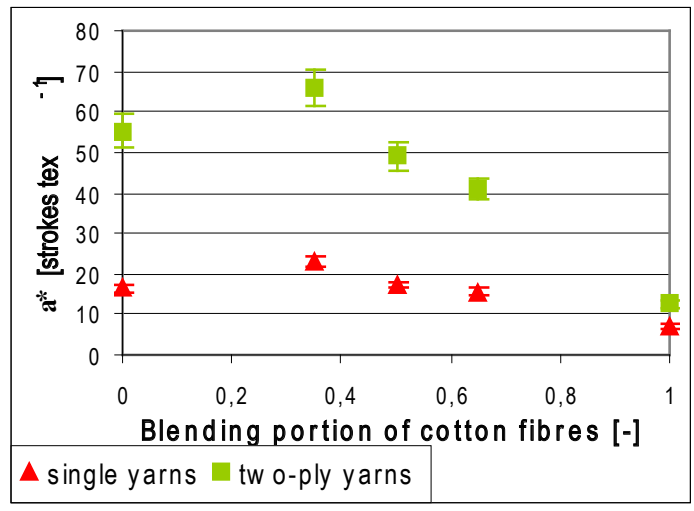

Figure 4. Comparison of mechanical parameters and blending portion of cotton fibers. 
of mechanical parameters correspond with our expectation. It is evident, that two-ply yarns have higher tenacity, comparable elongation, and higher abrasion resistance than single yarns. Tenacity of yarns decreases in respect to increasing blending portion of stronger component (PP); and that after reaching critical blending portion, point increases. Higher portion of stiffer stronger polypropylene fiber in yarn leads to lower elongation. General conclusion for dependence of abrasion resistance on blending portion was not found. Only semi-linear function can be suitable for trend description. Blending portion $35 \mathrm{PP} / 65 \mathrm{CO}$ seems to be optimal from the point of view of abrasion resistance and other mechanical parameters.

Analysis of yarn diameters before and after abrasion shows interesting results. Percentage of yarn diameter change describes yarn's abrasion resistance in terms of weight loss. Diameter comparisons $(D-D a) / D 10^{2},\left(D-D a_{\min }\right) / D 10^{2}$, and $D-D a_{\max } / / D 10^{2}$ show, that the change due to abrasion is more significant in case of single yarn. Typical trend of yarn diameter change in terms of blending portion can be found only for comparison of mean diameters $D$ and $D a$. Diameter $D$ decreases if blending portion of PP fibers decreases. Diameter change increases if blending portion of PP fibers decreases. Diameter reduction due to abrasion is more obvious in case of single $100 \%$ CO yarn and is about $20 \%$ (for $100 \%$ PP only $10 \%)$. Two-ply yarns show lower reduction of yarn diameter and is in a range from $1 \%$ to $3 \%$. Evaluation of diameter $D$ and $D a_{\text {min }}$ indicates that $40 \%$ reduction of diameter in case of single and two-ply yarn is possible. Yarn diameter enlargement is not so significant and is maximally $8 \%$ of original diameter. Twodimensional ANOVA analysis (factors: blending portion, plying)

a

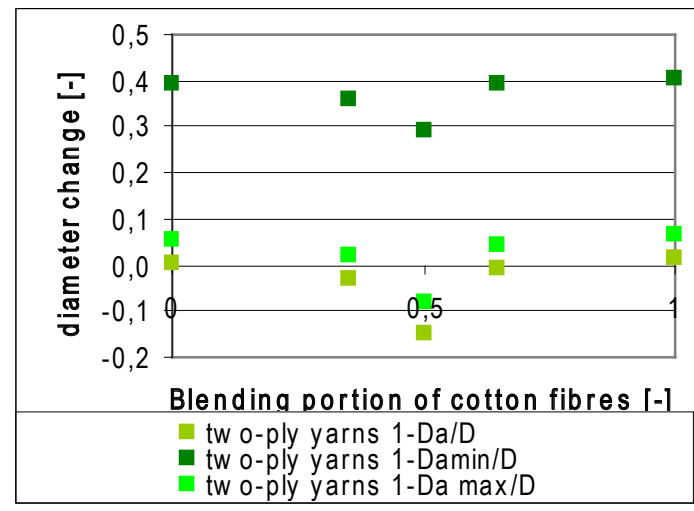

b

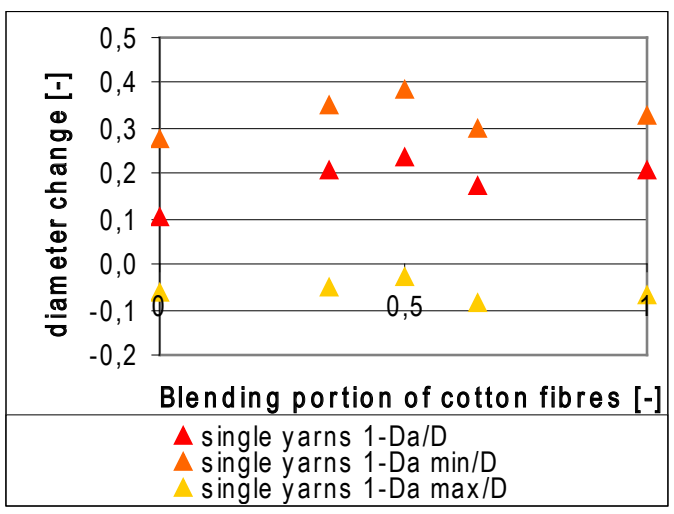

Figure 5. Comparison of yarn diameters before and after abrasion. confirms that plying technology is a significant factor, which influences mechanical parameters $F, e$, abrasion resistance a1, a2, and diameter change. Two-ply yarns made of $100 \% \mathrm{PP}$ and $65 \mathrm{PP} / 35 \mathrm{CO}$ are the best one in terms of strength, elongation, and abrasion resistance characteristics.

\section{Conclusion}

The main aim was to report about approaches to yarn resistance evaluation. Two sets of yarn were selected for experiment. Only the row of single and two-ply yarns was tested. The selected structure and mechanical parameters of fiber and yarn were analyzed. Zweigle G 552 was used for measuring yarn abrasion resistance. Number of strokes to yarn destruction and diameter change due to abrasion were observed. The generally known relationships were confirmed. Experimental results are in a good agreement with expectation. Increase of yarn count causes increase of yarn strength and abrasion resistance. Increase in number of twists is followed by increase of yarn strength and abrasion resistance. It is the reason of yarn elongation decrease. The influence of yarn count is in connection with number of fibers in yarn cross-section. Effect of twist is related to the level of fiber compactness. Two-ply yarn is more resistant to abrasion and is the reason of their using as warp yarn. Using of higher blending portion of a stiffer stronger component can improve yarn characteristics in terms of mechanical behavior and yarn abrasion. New approach to yarn abrasion evaluation was introduced. Interesting information was obtained thanks to comparison of yarn diameters before and after abrasion. Dimension of potentially thin and thick places is helpful for the assessment of weaving-ability. None of the parameters on its own provided a reliable method for establishing a definitive correlation between measurements made in laboratory and actual performance of yarns during weaving, because the process of mechanical yarn deformation on a loom is very complex and cannot be simulated absolutely during laboratory analysis.

\section{Acknowledgment}

This work was supported by the Textile Center II of Czech Ministry of Education $1 \mathrm{M} 0553$ and was published in AUTEX 2009 WORLD TEXTILE CONFERENCE May, 26-29, 2009 Çesme, Izmir, TURKEY.

\section{References}

[1] CTT yarn abrasion tester, Lawson Hemphil. http://www. lawsonhemphill.com/. Last Update 20.10. 2007.

[2] Goswami, B., C., Anandjiwala, R., D. and Hall, D. M.: Textile Sizing. Marcel Dekker, Inc., ISBN 0 8247-5053-5, USA - New York - Basel, (2004).

[3] Hearle, J., W., S., Grosberg, P., Backer, S.: Structural Mechanics of Fibers, Yarns and Fabrics. Wiley, New York 196.

[4] Křemenáková, D. and all: Internal Standards. Textile Research Centre Textile. Faculty of textile engineering. Technical University of Liberec 2004. 
[5] Operating instructions Zweigle G 567. www.zweigle.com. Last Update 20.10. 2007.

[6] Otsu, N.: A Threshold Selection Method from Gray-Level Histograms, IEEE Transactions on Systems, Man, and Cybernetics, Vol. 9, No. 1, 1979, pp. 62-66.

[7] Oxenham, W., Brzan, E. and Yu, C.: The abrasive properties of yarn. Department of Textile and Apparel, Technology and Management, College of Textiles, North Carolina State University web site.
[8] Webtester Reutlingen, ITV Denkendorf. www.itvdenkendorf.de. Last Update 20.10. 2007.

[9] Yarn abrasion tester Wira. www.wira.com. Last Update 20.10. 2007.

[10] Yarn on yarn abrasion test. Quantitative Measure of yarn durability. Tension Technology International, Technical Notes 18, January 2005. www.vectranfiber.com. Last Update 20.10. 2007. 